\title{
INDICATING THE MOST INFLUENTIAL MANUFACTURING FLEXIBILITY TYPES IN SCIENTIFIC RESEARCH
}

\author{
Gottfried Seebacher, Alpen-Adria University of Klagenfurt, Klagenfurt, AUSTRIA \\ Herwig Winkler, Alpen-Adria University of Klagenfurt, Klagenfurt, AUSTRIA
}

dx.doi.org/10.18374/EJM-14-1.2

\begin{abstract}
The flexibility of manufacturing systems has been discussed extensively in the scientific literature. Given the considerable heterogeneity and the growing variety of types and dimensions of manufacturing flexibility, the purpose of this paper is to explore the structure and origin of flexibility research, focusing on the types and dimensions of manufacturing flexibility. Additionally, the current paper aims at reducing the many flexibility types and dimensions to a limited set to provide a cleary arranged structure of the scientifically most important types and dimensions that affect the flexibility of manufacturing systems. Therefore, a sample of 153 internationally published papers containing a total of 5,060 citations that refer to 2,527 sources has been analysed to explore the most influential works and their impact on flexibility research. We evaluated the importance of the discussed flexibility types based on their citation frequency and attached greater value to more frequently cited and discussed flexibility types. Subsequently, we ranked the flexibility types to determine those that are most influential. Our results indicate that the key flexibility types of manufacturing systems are volume, process, and product flexibility.
\end{abstract}

Keywords: flexibility, manufacturing flexibility types, flexibility dimensions, flexibility management. 